\title{
Fluctuating Role of Parameters in the Analysis of the Continues and Discrete Version of a Susceptible-Incubated-Infected Model
}

\author{
Prasenjit Das $^{1}$, Debasis Mukherjee ${ }^{2}$, Kalyan Das ${ }^{3 *}$, A. Sabarmathi ${ }^{4}$ \\ ${ }^{1}$ Baikunthapur High School, Maipith, India \\ ${ }^{2}$ Department of Mathematics, Vivekananda College, Kolkata, India \\ ${ }^{3}$ Department of Mathematics, National Institute of Food Technology Entrepreneurship and Management, \\ HSIIDC Industrial Estate, Kundli, India \\ ${ }^{4}$ Department of Mathematics, School of Advanced Sciences, VIT University, Vellore, India \\ Email: jit_das2000@yahoo.com, debasis_mukherjee2000@yahoo.co.in, \{*daskalyan27, sabarmathi.a\}@gmail.com
}

Received March 16, 2012; revised April 15, 2012; accepted April 30, 2012

\begin{abstract}
The article concentrates on the role of fluctuating parameters for removable population from the incubated class in a susceptible-incubated-infected model. The discrete analogous of this model is also investigated. Conditions for local asymptotic stability are derived for both the disease free and endemic cases. Numerical simulations are performed to validate the theoretical results.
\end{abstract}

Keywords: Incubated Class; Stability; Schur-Cohn Criterion

\section{Introduction}

A rigorous study of mathematical models on biology shows that discrete-time models described by difference equations are more justified than the continuous-time models when the size of the population is rarely small or populations have non-overlapping generations. Further, epidemiological data for infectious diseases is collected in discrete from. Difference equation models give richer dynamics than continuous ones. Many authors [1-9] studied and analyzed the global stability, dynamics and chaotic behavior of various disease models. In the year 2009, G. Q. Sun [10] analyzed the Predator cannibalism can give rise to regular spatial pattern in a predator-prey system. In a recent works on disease model Dhar and Sharma [11] investigated the role of incubation period and showed that Hopf-bifurcation has occurred for certain threshold value of disease contact rate. In the study, they have considered logistic growth rate of susceptible populations. But in the present article we have analyzed the same model by considering constant recruitment rate of susceptible population along with its discrete version. The main thrust of our paper is to highlight the role of removable population from the incubated class. The paper is organized in the following manner. In Section 2 we present our model. The Section 3 deals with local behavior of the continuous-time model. Asymptotic behavior

${ }^{*}$ Corresponding author. of discrete version is carried out in Section 4. In Section 5 numerical examples and simulations are given and we round up the paper in Section 6.

\section{Model Equation}

Keeping in view that incubation period is a time period from exposure to onset of disease; we have considered that susceptible population instead of joining infected class directly will go through an intermediate class termed as incubated class. Let us consider total population is divided into three classes namely the susceptible class, incubated class and infected class. The continuous model system is described by the following equations:

$$
\begin{aligned}
& \frac{\mathrm{dS}}{\mathrm{dt}}=\mathrm{b}-\mathrm{dS}-\alpha \mathrm{SD}+\gamma \mathrm{D} \\
& \frac{\mathrm{dI}}{\mathrm{dt}}=\alpha \mathrm{SD}-\beta \mathrm{I} \\
& \frac{\mathrm{dD}}{\mathrm{dt}}=\beta_{1} \mathrm{I}-\delta \mathrm{D}
\end{aligned}
$$

Here $\mathrm{S}(\mathrm{t}), \mathrm{I}(\mathrm{t})$ and $\mathrm{D}(\mathrm{t})$ are the number of susceptible population, incubated population and infected population respectively at time $t$. The parameter $b$ is the recruitment rate of susceptible, and $d$ is its natural death rate. The parameter $\alpha$ represents the disease contact rate. $\gamma$ is the fraction of diseased population re- 
covery from disease while $\beta$ and $\delta$ respectively represent the total removable population from incubated class and from diseased class, which include death due to natural death and disease induced death. Further $\beta_{1}$ is the fraction of incubated class population that will go to the diseased class.

\section{Analysis of the Continuous Model}

\subsection{Equilibria}

The model system (1) has the following two equilibria: 1) Disease free equilibrium point $E_{1}(\bar{S}, 0,0)$ where $\overline{\mathrm{S}}=\frac{\mathrm{b}}{\mathrm{d}}$ and, 2) Endemic Equilibrium point $\mathrm{E}_{2}\left(\mathrm{~S}^{*}, \mathrm{I}^{*}, \mathrm{D}^{*}\right) \quad$ where $\mathrm{S}^{*}=\frac{\beta \delta}{\beta_{1} \alpha}, \quad \mathrm{I}^{*}=\frac{\delta\left(\mathrm{b} \beta_{1} \alpha-\beta \delta \mathrm{d}\right)}{\beta_{1} \alpha\left(\beta \delta-\gamma \beta_{1}\right)}$ and $\mathrm{D}^{*}=\frac{\mathrm{b} \beta_{1} \alpha-\beta \delta \mathrm{d}}{\alpha\left(\beta \delta-\gamma \beta_{1}\right)}$. Further $\mathrm{E}_{2}$ is feasible if $\frac{\gamma \beta_{1}}{\delta}<\beta<\frac{\mathrm{b} \beta_{1} \alpha}{\delta \mathrm{d}}$ or $\frac{\mathrm{b} \beta_{1} \alpha}{\delta \mathrm{d}}<\beta<\frac{\gamma \beta_{1}}{\delta}$.

\subsection{Local Stability}

We now state the stability behavior of the model system (1) at the endemic equilibrium point $E_{2}$.

Theorem1. The endemic equilibrium point $\mathrm{E}_{2}$ is stable if $\beta>\frac{\gamma \beta_{1}}{\delta}$ and is unstable if $\beta<\frac{\gamma \beta_{1}}{\delta}$.

Proof. The characteristic equation of the model system (1) at the point $\mathrm{E}_{2}$ is given by:

$$
\begin{aligned}
& \lambda^{3}+\mathrm{a}_{1} \lambda^{2}+\mathrm{a}_{2} \lambda+\mathrm{a}_{3}=0 \text { where } \mathrm{a}_{1}=\mathrm{d}+\alpha \mathrm{D}^{*}+\beta+\alpha, \\
& \mathrm{a}_{2}=(\beta+\delta)\left(\mathrm{d}+\alpha \mathrm{D}^{*}\right) \text { and } \mathrm{a}_{3}=\left(\alpha \mathrm{S}^{*}-\gamma\right) \alpha \mathrm{D}^{*} \beta_{1} .
\end{aligned}
$$

The result follows by the Routh-Hurwich criteria. Further it is important to note that when $\mathrm{E}_{1}$ is stable for $\beta>\frac{\mathrm{b} \beta_{1} \alpha}{\delta \mathrm{d}}$ then $\mathrm{E}_{2}$ is unstable. On the contrary while $\mathrm{E}_{1}$ is unstable for $\beta<\frac{\mathrm{b} \beta_{1} \alpha}{\delta \mathrm{d}}$ then $\mathrm{E}_{2}$ is stable.

\section{Analysis of Discrete Model}

In this section we investigate the dynamics of the discrete analogues of the continuous model (1). The model is described as follows:

$$
\begin{aligned}
& \mathrm{S}_{\mathrm{n}+1}=\mathrm{S}_{\mathrm{n}}+\mathrm{b}-\mathrm{dS}_{\mathrm{n}}-\alpha \mathrm{S}_{\mathrm{n}} \mathrm{D}_{\mathrm{n}}+\gamma \mathrm{D}_{\mathrm{n}} \\
& \mathrm{I}_{\mathrm{n}+1}=\mathrm{I}_{\mathrm{n}}+\alpha \mathrm{S}_{\mathrm{n}} \mathrm{D}_{\mathrm{n}}-\beta \mathrm{I}_{\mathrm{n}} \\
& \mathrm{D}_{\mathrm{n}+1}=\mathrm{D}_{\mathrm{n}}+\beta_{1} \mathrm{I}_{\mathrm{n}}-\delta \mathrm{D}_{\mathrm{n}}
\end{aligned}
$$

The fixed points of system (2) are as the follows: 1 ) $\mathrm{E}_{1}\left(\frac{\mathrm{b}}{\mathrm{d}}, 0,0\right)$ and 2) $\mathrm{E}_{2}\left(\mathrm{~S}^{*}, \mathrm{I}^{*}, \mathrm{D}^{*}\right)$ : where $\mathrm{S}^{*}=\frac{\beta \delta}{\beta_{1} \alpha}$,
$\mathrm{I}^{*}=\frac{\delta\left(\mathrm{b} \beta_{1} \alpha-\beta \delta \mathrm{d}\right)}{\beta_{1} \alpha\left(\beta \delta-\gamma \beta_{1}\right)} \quad$ and $\quad \mathrm{D}^{*}=\frac{\mathrm{b} \beta_{1} \alpha-\beta \delta \mathrm{d}}{\alpha\left(\beta \delta-\gamma \beta_{1}\right)} \quad$ provided $\frac{\gamma \beta_{1}}{\delta}<\beta<\frac{\mathrm{b} \beta_{1} \alpha}{\delta \mathrm{d}}$ or $\frac{\mathrm{b} \beta_{1} \alpha}{\delta \mathrm{d}}<\beta<\frac{\gamma \beta_{1}}{\delta}$.

We study asymptotic stability of the system of difference Equation (2) with the help of Schur-Cohn criterion [2]. That is, asymptotic stability of the system of difference equations can be found with the eigenvalues of Jacobian matrix of system (2).

\subsection{Stability Analysis of Fixed Point}

The Jacobian matrix of system (2) at the state variable is given by

$$
\mathrm{J}(\mathrm{S}, \mathrm{I}, \mathrm{D})=\left(\begin{array}{ccc}
-(\mathrm{d}+\alpha \mathrm{D}) & 0 & \gamma-\alpha \mathrm{S} \\
\alpha \mathrm{D} & -\beta & \alpha \mathrm{S} \\
0 & \beta_{1} & -\delta
\end{array}\right)
$$

We now state the local behavior of boundary fixed points.

Theorem 2. The fixed point $\mathrm{E}_{1}$ is locally stable if $\beta>\frac{\mathrm{b} \beta_{1} \alpha}{\delta \mathrm{d}}$ and is unstable if $\beta<\frac{\mathrm{b} \beta_{1} \alpha}{\delta \mathrm{d}}$.

Proof: The characteristic equation of the Jacobian matrix at $\mathrm{E}_{1}$ is $(\lambda+\mathrm{d})\left[\lambda^{2}+(\beta+\delta) \lambda+\beta \delta-\frac{\beta_{1} \alpha \mathrm{b}}{\mathrm{d}}\right]=0$ and the proof is obvious. Before stating the Theorem 3 we use the following theorem:

Theorem: Schur-Cohn criterion [2]: The zeros of the characteristic polynomial $\mathrm{p}(\lambda)=\lambda^{\mathrm{k}}+\mathrm{p}_{1} \lambda^{\mathrm{k}-1}+\cdots+\mathrm{p}_{\mathrm{k}}$ where the $p_{i}$ 's and only if the following results hold: are real numbers lie inside the unit disk if

1) $\mathrm{p}(1)>0,2)(-1)^{\mathrm{k}} \mathrm{p}(-1)>0$ and 3$)$ the $(\mathrm{k}-1) \times(\mathrm{k}-1)$ matrices

a)

$$
\beta_{\mathrm{k}-1}^{ \pm}=\left(\begin{array}{cccc}
1 & 0 & \cdots & 0 \\
\mathrm{p}_{1} & 1 & \cdots & 0 \\
\cdots & \cdots & \cdots & \cdots \\
\mathrm{p}_{\mathrm{k}-2} & \mathrm{p}_{\mathrm{k}-3} & \cdots & 1
\end{array}\right) \pm\left(\begin{array}{cccc}
0 & 0 & \cdots & \mathrm{p}_{\mathrm{k}} \\
0 & 0 & \cdots & \mathrm{p}_{\mathrm{k}-1} \\
\cdots & \cdots & \cdots & \cdots \\
\mathrm{p}_{\mathrm{k}} & \mathrm{p}_{\mathrm{k}-1} & \cdots & \mathrm{p}_{2}
\end{array}\right)
$$

b) a real positive innerwise.

Theorem 3. The fixed point $\mathrm{E}_{2}$ is asymptotically stable if the condition $\left|\mathrm{p}_{2}-\mathrm{p}_{1} \mathrm{p}_{3}\right|<1-\mathrm{p}_{3}^{2}$ is satisfied.

Proof: The characteristic equation of the above Jacobian matrix at $\mathrm{E}_{2}$ is $\lambda^{3}+\mathrm{p}_{1} \lambda^{2}+\mathrm{p}_{2} \lambda+\mathrm{p}_{3}=0$ where, $\mathrm{p}_{1}=\mathrm{a}_{1}-3, \mathrm{p}_{2}=3-2 \mathrm{a}_{1}+\mathrm{a}_{2}$ and $\mathrm{p}_{3}=\mathrm{a}_{3}$. The result is followed by the application of Schur-Cohn criterion.

\section{Numerical Examples and Simulations}

We now provide some examples to discuss the dynamics of the system with some parameter values. Choosing $b=$ 
$0.4, \mathrm{~d}=0.05, \alpha=0.02, \gamma=0.01, \beta=0.02, \beta_{1}=0.01$, and $\delta=0.02$ we observe that the system (1) has two equilibrium points namely the disease free equilibrium point $(8,0,0)$ and the endemic equilibrium point $(2,20$, 10). From numerical simulations we observe that the endemic equilibrium is stable for both the continuous case (Figure 1(a)) and in discrete case (Figure 1(b)).

Considering the parameter values $\mathrm{b}=0.25, \mathrm{~d}=1, \alpha$
$=0.5, \gamma=0.5, \beta=0.1, \beta_{1}=0.3$ and $\delta=1$, we observe that the disease free fixed point $(0.25,0,0)$ is stable for both the continuous time model (Figure 2(a)) and discrete time model (Figure 2(b)).

Considering the parameter values $\mathrm{b}=0.25, \mathrm{~d}=1, \alpha$ $=1, \gamma=0.5, \beta=0.3, \beta_{1}=1$ and $\delta=1$. We get the endemic fixed point $(0.3,0.25,0.25)$. From numerical simulations (see Figures 3(a) and (b)) it is observed that

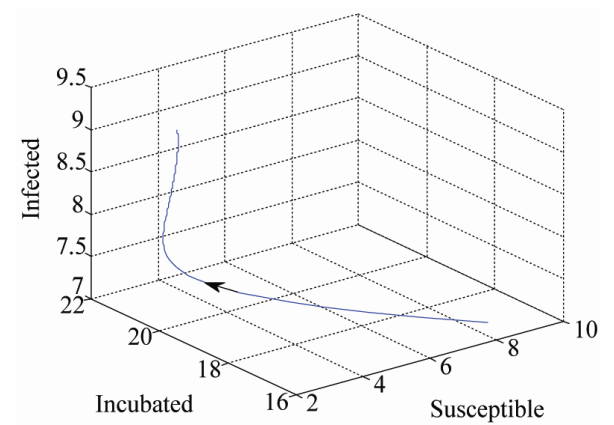

(a)

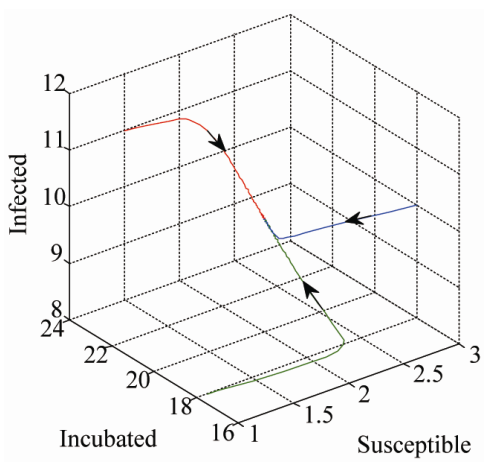

(b)

Figure 1. (a) Stable Endemic equilibrium for continuouscase; (b) Stable Endemic equilibrium for discrete case.

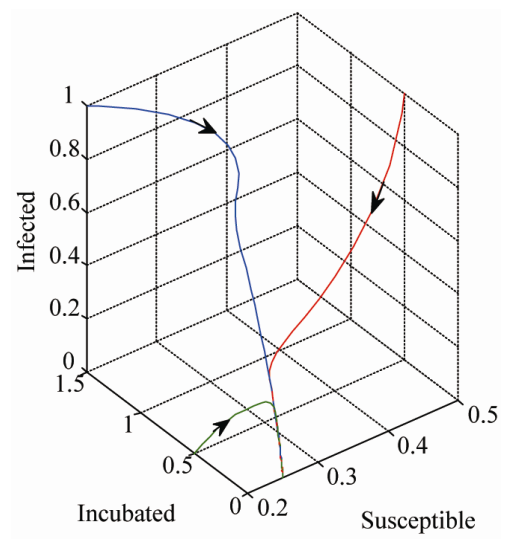

(a)

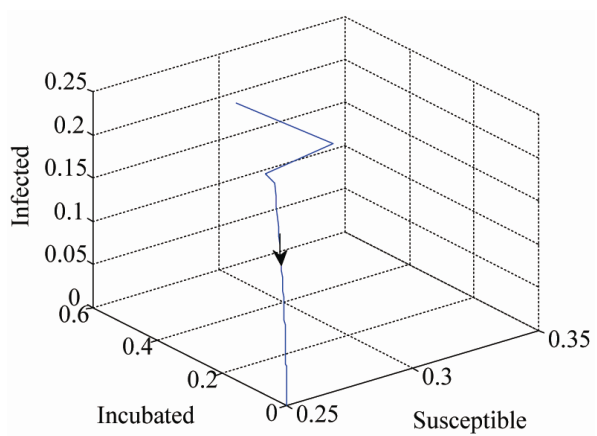

(b)

Figure 1. (a) Disease free stable fixed point for continuous model; (b) Disease free stable fixed point for discrete model.

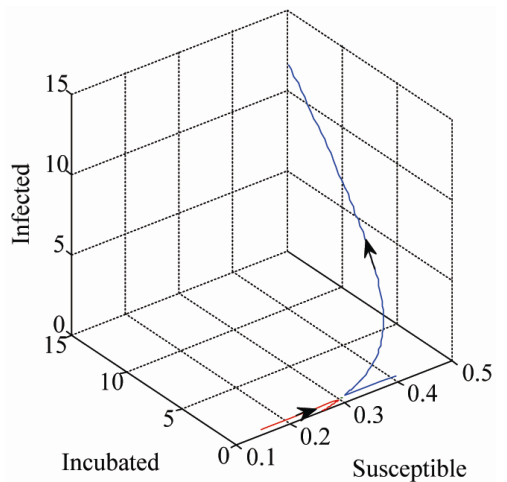

(a)

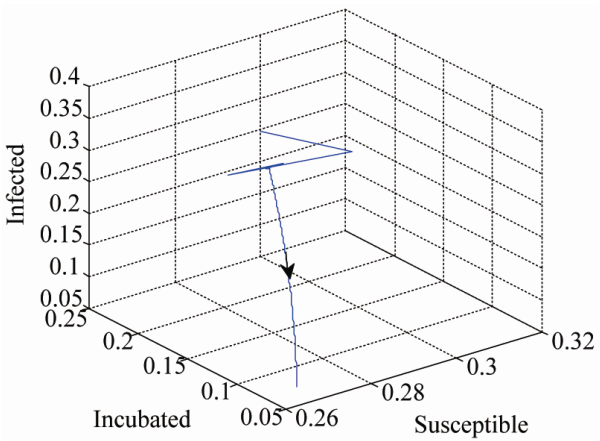

(b)

Figure 2. (a) Unstable endemic equilibrium point for continuous time model; (b) Unstable endemic equilibrium point for discrete time model. 
the endemic equilibrium point is unstable for both the continuous time model and discrete time model.

\section{Discussion}

The analytical results and numerical findings of the paper suggest the removable rate of population from incubated class $(\beta)$ which plays an important role on the dynamics of the system. The disease free equilibrium approaches to the endemic equilibrium when $\beta$ is above a certain threshold value and on the contrary the endemic equilibrium approaches to the disease free equilibrium below this thresh- old of $\beta$. So the disease outbreak can be under control with the parameter $\beta$. On the other hand in paper [11] authors have shown that the disease transfer rate from susceptible to incubated population as a bifurcation parameter. In brief, we can conclude that though the disease is endemic in nature initially, still in the long run, it would be possible to control the disease and even if it may also be eradicated from the society based on the number of removable population from incubated class.

\section{Acknowledgements}

Authors are thankful to the reviewers for their valuable comments and suggestions to improve this paper.

\section{REFERENCES}

[1] M. J. Stutzer, "Chaotic Dynamics and Bifurcation in a Micro Model," Journal of Economic Dynamics and Control, Vol. 2, 1980, pp. 353-376. doi:10.1016/0165-1889(80)90070-6

[2] R. L. Deveney, "An Introduction to Chaotic Dynamical Systems,” Addison-Wisely Publishing Company Inc., Boston, 1986.
[3] H. Inaba, "Threshold and Stability Results for an AgeStructured Epidemic Model," Journal of Mathematical Biology, Vol. 28, No. 4, 1990, pp. 411-34. doi:10.1007/BF00178326

[4] M. Y. Li and J. S. Muldowney, "Global Stability for the SEIR Model in Epidemiology,” Mathematical Bioscience, Vol. 125, No. 2, 1995, pp. 155-164. doi:10.1016/0025-5564(95)92756-5

[5] R. C. Hilborn, "Chaos and Non-Linear Dynamics: An Introduction for Scientist and Engineers,” 2nd Edition, Oxford University Press, Oxford, 2000.

[6] S. Elaydi, “An Introduction to Difference Equation,” Springer-Verlag, Berlin, 2005.

[7] A. D’Onofrio, P. Manfredi and E. Salinelli, "Vaccinating Behaviour, Information, and the Dynamics of SIR Vaccine Preventable Diseases," Theoretical Population Biology, Vol. 71, No. 3, 2007, pp. 301-317. doi:10.1016/j.tpb.2007.01.001

[8] W. M. Schaffer and T. V. Bronnikova, "Parametric Dependence in Model Epidemics,” Journal of Bioogical Dynamics, Vol. 1, No. 2, 2007, pp. 183-195. doi:10.1080/17513750601174216

[9] F. M. Hilker, H. Malchow, M. Langlais and S. V. Petrovskii, "Oscillations and Waves in a Virally Infected Plankton System, Transition from Lysogeny to Lysis," Journal of Ecological Complexity, Vol. 3, No. 3, 2006, pp. 200-208.

[10] G. Q. Sun, G. Zhang, Z. Jin and L. Li, "Predator Cannibalism can Give Rise to Regular Spatial Pattern in a Predator-Prey System,” Nonlinear Dynamics, Vol. 58, No. 1, 2009, pp. 75-84. doi:10.1007/s11071-008-9462-z

[11] J. Dhar and A. K. Sharma, "The Role of the Incubation Period in a Disease Model," Applied Mathematics ENotes, Vol. 9, 2009, pp. 146-153. 